\title{
RECENT PROGRESS OF INVERSE SCATTERING THEORY ON NON-COMPACT MANIFOLDS
}

\author{
HIROSHI ISOZAKI, YAROSLAV KURYLEV, AND MATTI LASSAS \\ Dedicated to Gunther Uhlmann on the occasion of his 60th birthday
}

\begin{abstract}
We give a brief survey for the recent development of inverse scattering theory on non-compact Riemannian manifolds. The main theme is the reconstruction of the manifold and the metric from the scattering matrix.
\end{abstract}

\section{INTRODUCTION}

Scattering theory for waves in classical or quantum physics has a long history, and nowadays there is an abundance of works devoted to the forward and inverse problems of potential scattering for Schrödinger equations, and obstacle scattering for wave equations. Much less is known, however, about the inverse scattering on manifolds, where the main theme is the reconstruction of the manifold itself and its Riemannian metric from the scattering matrix associated with the LaplaceBeltrami operator. We have been working on this subject by choosing typical examples possessing characteristic features of the problem. The aim of this paper is to present a birds-eye view based on the results we have obtained so far.

In $\S 2$, we discuss a basic framework of our issue. Scattering of waves is a timedependent phenomenon, however, it is a consequence of properties of the continuous spectrum of the underlying Laplacian. Therefore, we formulate the problem mainly from the stationary view point with emphasis on the solution space of the Helmholtz equation. In $\S 3$, we explain the outline of our theory for the forward and inverse problems ignoring the detailed assumptions of the metric. In $\S 4$, we pick up four metrics we have already studied. They are well-known standard examples, and, viewed from the growth order of volume near infinity, range from an exponentially growing case to an exponentially decaying case. In $\S 5$, we devote ourselves to the case of 2-dim. arithmetic surface and discuss the inverse scattering from cusp.

In $\S 6$, we mention some recent results for metrics with intermediate behavior.

Due to the lack of space, we cannot talk about all of the important works. For example, 12, 11, [13, [50, 54, 55, are dealing with problems related to ours from different view points.

\section{REVIEW OF FORWARD AND INVERSE PROBLEMS}

2.1. Time-dependent scattering problem. Let us consider $\mathbb{R}^{n}, n>1$, with a Riemannian metric $G=g_{i j} d x^{i} d x^{j}$, where we use the Einstein summation convention of omitting sum signs. We assume that asymptotically, when $|x| \rightarrow \infty$, $g_{i j}(x) \simeq \delta_{i j}$, see e.g. (4.1). How can one recover the metric tensor $g_{i j}$ from some

1991 Mathematics Subject Classification. Primary 35R30; Secondary 81U40. 
"physical" observations? One way is to consider wave motions associated with this metric:

$$
\partial_{t}^{2} v(t, x)=\Delta_{G} v(t, x),
$$

where $\Delta_{G}$ is the (negative) Laplace-Beltrami operator associated with $G$. In the remost past (i.e. $t \rightarrow-\infty$ ) and in the remote future (i.e. $t \rightarrow \infty$ ), the solution to (2.1) behaves like $v_{ \pm}$satisfying $\partial_{t}^{2} v_{ \pm}=\Delta v_{ \pm}, \Delta$ being the standard Euclidean Laplacian on $\mathbb{R}^{n}$. The mapping assigning the far-field pattern of $v_{-}$to that of $v_{+}$is called the scattering operator. It is a common belief that, under natural situations, this scattering operator determines the original physical system, i.e. the metric, at least up to changes of coordinates. In other words, from the observation of all far-field patterns of waves at infinity, one can reconstruct the metric $G$. For elliptic inverse boundary value problems on compact or non-compact Riemannian manifolds there are also non-uniqueness results; one can use the fact that the measurements are invariant in diffeomorphisms of the manifold to create counterexamples for the uniqueness of inverse problems and even invisibility results, see [18, 19, 35, 44 and the references in [20, 21]

Another way is to use the wave motion of quantum mechanical particles, in which case we use the Schrödinger equation

$$
i \partial_{t} v(t, x)=\Delta_{G} v(t, x),
$$

instead of (2.1). (See e.g. 56] or 61]). Both of these time-dependent scattering problems are reformulated in the same stationary (time-independent) picture.

2.2. Stationary scattering theory. Consider a time-periodic motion of (2.1), i.e. put $v(t, x)=e^{-i k t} u(x)$ in (2.1). Then we get

$$
\left(-\Delta_{G}-k^{2}\right) u(x)=0, \quad k>0 .
$$

Since all solution of the Helmholtz equation (2.3) with unperturbed Laplacian, $\Delta$, can be written as superpositions of plane waves and taking into account that $g_{i j} \simeq \delta_{i j}$, we seek a solution of (2.3) admitting the following asymptotic expansion

$$
u(x) \simeq e^{i k \omega \cdot x}+\frac{e^{i k r}}{r^{(n-1) / 2}} a(\hat{x}, \omega ; k), \quad \hat{x}=x / r, \quad \text { as } \quad r=|x| \rightarrow \infty,
$$

where $\omega \in S^{n-1}$. The 2 nd term of the right-hand side represents the scattered spherical wave. In the case of quantum mechanics, for a given beam of particles with initial direction $\omega,|a(\theta, \omega ; k)|^{2}$ is proportional to the number of particles scattered to the $\theta$-direction. This is the physical quantity observed in the experiment. Let $A(k): L^{2}\left(S^{n-1}\right) \rightarrow L^{2}\left(S^{n-1}\right)$ be the integral operator with the kernel $a(\hat{x}, \omega ; k)$. By a suitable choice of the constant $C(k), S(k)=I-C(k) A(k)$ is a unitary operator on $L^{2}\left(S^{n-1}\right)$, which is the well-known Heisenberg's S-matrix. The above mentioned scattering operator in the time-dependent formulation is written in terms this Smatrix.

2.3. Geometric scattering theory. Let us look at the stationary scattering theory from a geometrical view point. Consider the restriction of the Fourier transform on $S^{n-1}$ :

$$
\left(\mathcal{F}_{0}(k)^{*} \varphi\right)(x)=\int_{S^{n-1}} e^{i k \omega \cdot x} \varphi(\omega) d \omega, \quad k>0 .
$$


This is sometimes called the Herglotz integral or the Poisson integral. It is the eigenoperator of $-\Delta$ in the sense that it satisfies the equation

$$
\left(-\Delta-k^{2}\right) \mathcal{F}_{0}(k)^{*} \varphi=0 .
$$

By the stationary phase method, it admits the asymptotic expansion:

$$
\left(\mathcal{F}_{0}(k)^{*} \varphi\right)(x) \simeq C_{+}(k) \frac{e^{i k r}}{r^{(n-1) / 2}} \varphi(\hat{x})+C_{-}(k) \frac{e^{-i k r}}{r^{(n-1) / 2}} \varphi(-\hat{x}), \quad r=|x| \rightarrow \infty .
$$

Let us note that the spherical waves $e^{ \pm i k r} / r^{(n-1) / 2}$ appear in the asymptotic expansion of the Green functions $G_{0}^{( \pm)}(x, y ; k)$ of $-\Delta-k^{2}$ in $\mathbb{R}^{n}$ :

$$
\int_{\mathbb{R}^{n}} G_{0}^{( \pm)}(x, y ; k) f(y) d y \simeq \widetilde{C}_{ \pm}(k) \frac{e^{ \pm i k r}}{r^{(n-1) / 2}} \widehat{f}( \pm k \hat{x}),
$$

where $\widehat{f}(\xi)$ is the Fourier transform of $f(y)$. The unit sphere $S^{n-1}$ can be regarded as a boundary at infinity of $\mathbb{R}^{n}$. Therefore, for $\mathbb{R}^{n}$ with Euclidean metric, one can associate the manifold at infinity, $S^{n-1}$, and the integral transform on it, $\mathcal{F}_{0}(k)^{*}$. It is an eigenoperator of $-\Delta$ and has the asymptotic expansion (2.5).

The stationary scattering theory asserts that these properties are transferred to $\mathbb{R}^{n}$ with metric $G$. Namely, one can associate a generalized eigenoperator $\mathcal{F}(k)^{*}$ on $L^{2}\left(S^{n-1}\right)$ satisfying

$$
\left(-\Delta_{G}-k^{2}\right) \mathcal{F}(k)^{*} \varphi=0, \quad \text { for all } k^{2} \in \sigma_{c}\left(-\Delta_{G}\right),
$$

and admitting the asymptotic expansion (2.5) with $\mathcal{F}_{0}(k)^{*}$ replaced by $\mathcal{F}(k)^{*}$. Moreover, by introducing a Banach space $\mathcal{B}^{*}$ by

$$
\mathcal{B}^{*} \ni u \Longleftrightarrow \sup _{R>1} \frac{1}{R} \int_{|x|<R}|u(x)|^{2} d x<\infty,
$$

one can show that

$$
\mathcal{N}(k):=\left\{u \in \mathcal{B}^{*} ;\left(-\Delta_{G}-k^{2}\right) u=0\right\}=\mathcal{F}(k)^{*}\left(L^{2}\left(S^{n-1}\right)\right),
$$

and that for any $\varphi_{-} \in L^{2}\left(S^{n-1}\right)$, there exist unique $u \in \mathcal{N}(k)$ and $\varphi_{+} \in L^{2}\left(S^{n-1}\right)$ satisfying

$$
u(x) \simeq C_{+}(k) \frac{e^{i k r}}{r^{(n-1) / 2}} \varphi_{+}(\hat{x})+C_{-}(k) \frac{e^{-i k r}}{r^{(n-1) / 2}} \varphi_{-}(\hat{x}), \quad r=|x| \rightarrow \infty .
$$

Here, by $f \simeq g$, we mean

$$
\lim _{R \rightarrow \infty} \frac{1}{R} \int_{|x|<R}|f(x)-g(x)|^{2} d x=0 .
$$

The mapping

$$
S_{\text {geo }}(k): L^{2}\left(S^{n-1}\right) \ni \varphi_{-} \rightarrow \varphi_{+} \in L^{2}\left(S^{n-1}\right)
$$

is unitary. In fact, it is related with the above S-matrix as follows:

$$
S_{g e o}(k)=J S(k),
$$

where $J \phi(\omega)=\phi(-\omega)$.

Motivated by the above, let us give an overview how a geometric scattering theory on a Riemannian manifold can be formulated. We are given a non-compact Riemannian manifold $\mathcal{M}$ having the boundary at infinity $M$, which is a compact 
Riemannian manifold of dimension $n-1$. We then construct an operator $\mathcal{F}(k)^{*}$ : $L^{2}(M) \rightarrow L_{\text {loc }}^{2}(\mathcal{M})$ such that

$$
\left(-\Delta_{G}-k^{2}\right) \mathcal{F}(k)^{*} \varphi=0, \quad \text { for all } k^{2} \in \sigma_{c}\left(-\Delta_{G}\right),
$$

with the property that, by a suitable choice of the Banach space $\mathcal{B}^{*}$ of functions in $\mathcal{M}$,

$$
\mathcal{N}(k):=\left\{u \in \mathcal{B}^{*} ;\left(-\Delta_{G}-k^{2}\right) u=0\right\}=\mathcal{F}(k)^{*}\left(L^{2}(M)\right) .
$$

Moreover, there exist functions $w_{ \pm}(k) \in \mathcal{B}^{*}$ such that for any $\varphi_{-} \in L^{2}(M)$ there exist unique $u \in \mathcal{N}(k)$ and $\varphi_{+} \in L^{2}(M)$ admitting the asymptotic expansion

$$
u \simeq w_{+}(k) \varphi_{+}+w_{-}(k) \varphi_{-},
$$

and the mapping

$$
S_{g e o}(k): L^{2}(M) \ni \varphi_{-} \rightarrow \varphi_{+} \in L^{2}(M)
$$

is unitary. We then formulate our inverse problem as follows : Reconstruct the manifold $\mathcal{M}$ and its metric from the knowledge of the $S$-matrix $S_{g e o}(k)$.

2.4. Inverse scattering. The inverse potential scattering on the half-line was solved in 1950's by Gel'fand-Levitan and Marchenko [17, 46]. The multi-dimensional extension was proposed by the seminal work of Faddeev [16. We do not enter into the detailed exposition here (see e.g. 30), however, let us emphasize that the so called CGO solution of the Schrödinger equation introduced by Calderón and implemented into a powerfull tool by Sylvester and Uhlmann [58] (in fact, it was done independently of Faddeev) stimulated the works of Nachman [53] and KhenkinNovikov [36] to bring the progress in the multi-dimensional inverse problem.

As for our inverse scattering problem, we use the boundary control (BC) method. It is now regarded as an established theory for the inverse spectral problem, however, a long time was necessary to settle the whole idea. The precursor of the BC method appeared in the work of M. G. Krein on the 1-dimensional wave equation 37, 38. Although there is a similarity of Krein's theory to that of Gel'fandLevitan-Marchenko, his idea is based on the hyperbolic nature of the wave equation, i.e. finite velocity of the wave propagation and the notion of the domain of influence. However, by passing to the Fourier transform in $t$, he used it in the form of the analyticity properties with respect to the spectral parameter. Blagovestcenskii 9 analyzed Krein's idea, using the finite velocity and controllability of the filled domain, to derive a Volterra-type equation for unknown functions. In [10, he also found a crucial idea of evaluating the inner product of waves with given data on the boundary by the spectral data. These ideas were then extended to the multi-dimensional case by the works of Belishev and Belishev-Blagovestcenskii [3], 5. Belishev and Kurylev [7] used the BC method to solve the Gel'fand inverse problem on compact Riemannian manifolds. We must also mention that the step of controllability was completed by Tataru [59], which gave a final form for Holmgren's uniqueness theorem. The BC method can also be used to reconstruct non-smooth manifolds [2, 42] or solve inverse problems for the heat and Schrödinger equations on Riemannian manifolds 33. For the more detailed history of the BC method and the related reconstruction methods, see [4, 8, 39, 32. 


\section{BIRD'S-EYE VIEW OF THE INVERSE SCATTERING PROBLEM FOR THE METRIC}

We shall discuss here "typical" results which we expect to hold. Therefore, we state Theorems and Lemmas "formally" without specfying the detailed assumptions.

3.1. General framework. We consider an $n$-dimensional, non-compact, connected, Riemannian manifold $\mathcal{M}$ with the following properties :

$$
\mathcal{M}=\mathcal{K} \cup \mathcal{M}_{1} \cup \cdots \cup \mathcal{M}_{N},
$$

where $\mathcal{K}$ is relatively compact, and $\mathcal{M}_{i}$ 's (called ends) are non-compact. We allow $\mathcal{K}$ to have an arbitrarily metric, however, each $\mathcal{M}_{i}$ is assumed to be diffeomorphic to $(1, \infty) \times M_{i}$, where $M_{i}$ is a compact $(n-1)$-dimensional manifold endowed with a metric $h_{i}=\left(h_{i}\right)_{\alpha \beta}(y) d y^{\alpha} d y^{\beta}$. Let us denote points of $(1, \infty) \times M_{i}$ by $(r, y)$. We also assume that on each end $\mathcal{M}_{i}$, the metric $G$ is asymptotically equal to the warped product:

$$
G \sim(d r)^{2}+\rho_{i}(r)\left(h_{i}\right)_{\alpha \beta}(y) d y^{\alpha} d y^{\beta}, \quad \text { as } \quad r \rightarrow \infty,
$$

with some positive function $\rho_{i}(r) \in C^{\infty}(\mathbb{R})$. We assume $\mathcal{M}$ to be complete, hence $-\left.\Delta_{G}\right|_{C_{0}^{\infty}(\mathcal{M})}$ is essentially self-adjoint. For the sake of simplicity, assume that $\sigma_{c}\left(-\Delta_{G}\right)=\left[E_{0}, \infty\right)$, and put

$$
H=-\Delta_{G}-E_{0} .
$$

The properties of the continuous spectrum of $H$ are determined by those of ends $\mathcal{M}_{i}$. Therefore, we reduce our analysis to a suitable model space, whose choice depends largely on the nature of $\rho_{i}(r)$, and shall be discussed separately.

3.2. Analysis on the model space. To fix our assumptions, we consider the case when the boundary $\partial \mathcal{M}$ is empty. Note that, however, one could consider, in a similar manner, also the case when $\partial \mathcal{M} \neq \emptyset$ with Neumann, Dirichlet or other self-adjoint boundary conditions. Given $\rho(r) \in C^{\infty}(\mathbb{R})$, we take $\mathcal{M}_{0}=I_{0} \times M_{0}$, where $I_{0}=\left(c_{0}, \infty\right)$ is an open interval such that $c_{0}<1$, equipped with the metric $G_{0}=(d r)^{2}+\rho(r) h_{0}$. By imposing the Neumann boundary condition at $r=c_{0}$, we assume that the Laplace-Beltrami operator $H_{0}=-\Delta_{G_{0}}$ on $\mathcal{M}_{0}$ is self-adjoint in $L^{2}\left(I_{0}, L^{2}\left(M_{0}\right) ; \rho(r)^{(n-1) / 2} d r\right)$. Assume that $\sigma_{c}\left(-\Delta_{G_{0}}\right)=\left[E_{0}, \infty\right)$, and put $H_{0}=$ $-\Delta_{G_{0}}-E_{0}, R_{0}(z)=\left(H_{0}-z\right)^{-1}$. Let us call $H_{0}$ the free operator on $\mathcal{M}_{0}$. Our first task is to prove the limiting absorption principle, which is going to be explained below.

Let $A=\int_{\mathbb{R}} \lambda d E_{A}(\lambda)$ be a self-adjoint operator on a Hilbert space $\mathcal{H}$. Then for $\lambda \in \sigma(A),(A-\lambda)^{-1}$ does not exist. However, for $\lambda \in \sigma_{c}(A)$, sometimes one can prove the existence of the limit

$$
(A-\lambda \mp i 0)^{-1}=\lim _{\epsilon \rightarrow 0}(A-\lambda \mp i \epsilon)^{-1} \in \mathbf{B}\left(\mathcal{H}_{+} ; \mathcal{H}_{-}\right),
$$

where $\mathbf{B}(X ; Y)$ means the set of all bounded operators from a Banach space $X$ to a Banach space $Y$, and $\mathcal{H}_{ \pm}$are suitable Banach spaces skirting $\mathcal{H}$ :

$$
\mathcal{H}_{+} \subset \mathcal{H} \subset \mathcal{H}_{-}
$$

with inclusions dense and continuous, and $\mathcal{H}_{-}=\left(\mathcal{H}_{+}\right)^{*}$ through the inner product of $\mathcal{H}$. This is called the limiting absorption principle, abbreviated as LAP. If the 
LAP is proved on an interval $I \subset \sigma_{c}(A)$, by Stone's formula, we have

$$
E_{A}(I) \mathcal{H} \subset \mathcal{H}_{a c}(A),
$$

where the absolutely continuous subspace $\mathcal{H}_{a c}(A)$ is defined by

$$
\mathcal{H}_{a c}(A) \ni u \Longleftrightarrow\left(E_{A}(\lambda) u, u\right) \text { is absolutely continuous w.r.t. } d \lambda \text {. }
$$

In all examples we have already treated, the space $\mathcal{H}_{-}$is defined as follows.

$$
\mathcal{H}_{-} \ni u \Longleftrightarrow \sup _{R>1} \frac{1}{R} \int_{c_{0}<r<R}\|u(r)\|_{L^{2}\left(M_{0}\right)}^{2} \rho(r)^{(n-1) / 2} d r<\infty .
$$

This is a dual space of some Banach space $\mathcal{B}$ using a dyadic decomposition of the manifold $\mathcal{M}_{0}$, and is denoted by $\mathcal{B}^{*}$ below. To prove LAP, usually we need to avoid the end points of $\sigma_{c}(H)$, the eigenvalues embedded in the continuous spectrum, and thresholds (the energies at which the nature of scattering changes). Let us call the set of these points the exceptional set, and denote it by $\mathcal{E}_{0}$. This is shown to be discrete with possible accumulation points 0 and on thresholds.

Our next task is to analyse the asymptotic behavior of the resolvent $R_{0}(\lambda \pm i 0)$ as $r \rightarrow \infty$. For $f, g \in \mathcal{H}_{-}$we use the notation $f \simeq g$ to denote

$$
f \simeq g \Longleftrightarrow \lim _{R \rightarrow \infty} \frac{1}{R} \int_{c_{0}<r<R}\|f(r)-g(r)\|_{L^{2}\left(M_{0}\right)}^{2} \rho(r)^{(n-1) / 2} d r=0 .
$$

Then for any $k>0$ such that $k^{2} \notin \mathcal{E}_{0}$, there exist bounded operators $\mathcal{F}_{0}^{( \pm)}(k) \in$ $\mathbf{B}\left(\mathcal{B} ; L^{2}\left(M_{0}\right)\right)$ and functions $w_{ \pm}(k) \in \mathcal{B}^{*}$ such that for $f \in \mathcal{B}$,

$$
R_{0}\left(k^{2} \pm i 0\right) f \simeq w_{ \pm}(k) \mathcal{F}_{0}^{( \pm)}(k) f .
$$

3.3. Gluing the estimates for ends. Having established the LAP and the asymptotic expansion (3.4) on each end, we need to glue them together to obtain global results. Let $\left\{\chi_{j}\right\}_{j=0}^{N}$ be a partition of unity on $\mathcal{M}$ such that $\sum_{j=0}^{N} \chi_{j}=1$, and, for $j=1, \cdots, N, \operatorname{supp}\left(\chi_{j}\right) \subset \mathcal{M}_{j}, \chi_{j}(r)=1$ if $r>2$. We take $\tilde{\chi}_{j} \in C^{\infty}\left(\mathcal{M}_{j}\right)$ such that $\operatorname{supp}\left(\tilde{\chi}_{j}\right) \subset \mathcal{M}_{j}$ and $\tilde{\chi}_{j}=1$ on $\operatorname{supp} \chi_{j}$. Let $\mathcal{H}_{\text {free }(j)}$ be the free operator on $\mathcal{M}_{j}$ and assume, for simplicity, that for all $j=1, \ldots, N$, we have $\sigma_{c}\left(\mathcal{H}_{\text {free }(j)}\right)=\left[E_{0}, \infty\right)$. Then we put $R_{\text {free }(j)}(z)=\left(H_{\text {free }(j)}-z\right)^{-1}$ and

$$
\begin{gathered}
A(z)=\sum_{j=1}^{N} A_{j}(z) \tilde{\chi}_{j}, \\
A_{j}(z)=\left[H, \chi_{j}\right] R_{\text {free }(j)}(z)+\chi_{j}\left(H-H_{\text {free }(j)}\right) \tilde{\chi}_{j} R_{\text {free }(j)}(z) .
\end{gathered}
$$

Then we have

$$
R(z)=\sum_{j=1}^{N} \chi_{j} R_{\text {free }(j)}(z) \widetilde{\chi}_{j}+R(z)\left(\chi_{0}-A(z)\right) .
$$

This formula and the perturbation technique enable us to prove LAP for $H$. Let $\mathcal{E}=\left(\cup_{j=1}^{N} \mathcal{E}_{j}\right) \cup\left(\sigma_{p}(H) \cap[0, \infty)\right), \mathcal{E}_{j}$ being the exceptional set for $H_{\text {free }(j)}$. One can show that $\mathcal{E}$ is discrete with possible accumulation points 0 and on thresholds of $H_{\text {free }(j)}$. We define the space $\mathcal{B}^{*}$ by gluing $\mathcal{B}_{j}{ }^{*}$ using the partition of unity, namely,

$$
\mathcal{B}^{*}=\left\{u \in L_{l o c}^{2}(\mathcal{M}): \chi_{j} u \in \mathcal{B}_{j}^{*}\right\} .
$$

Theorem 3.1. For $\lambda \in[0, \infty) \backslash \mathcal{E}$, there exists a limit $R(\lambda \pm i 0) \in \mathbf{B}\left(\mathcal{B} ; \mathcal{B}^{*}\right)$. 
By taking the adjoint in (3.5), we can derive the asymptotic expansion of the resolvent on each end:

$$
R\left(k^{2} \pm i 0\right) f \simeq w_{j}^{( \pm)}(k) \mathcal{F}_{j}^{( \pm)}(k) f, \quad f \in \mathcal{B} .
$$

We now introduce the $L^{2}$-space over the manifold at infinity of $\mathcal{M}$ :

$$
\mathbf{h}_{\infty}=\oplus_{j=1}^{N} L^{2}\left(M_{j}\right) .
$$

We also put

$$
\begin{gathered}
\left.\mathcal{F}^{( \pm)}(k)=\left(\mathcal{F}_{1}^{( \pm)}(k), \cdots, \mathcal{F}_{N}^{( \pm)} k\right)\right) \in \mathbf{B}\left(\mathcal{B} ; \mathbf{h}_{\infty}\right), \\
\widehat{\mathcal{H}}=L^{2}\left((0, \infty), \mathbf{h}_{\infty} ; d k\right) .
\end{gathered}
$$

Here by $L^{2}(I, \mathbf{h} ; d k)$ with given auxiliary Hilbert space $\mathbf{h}$, we mean the Hilbert space of all $\mathbf{h}$-valued $L^{2}$-functions on an interval $I$ with respect to the measure $d k$.

Theorem 3.2. (1) The operator $\mathcal{F}^{( \pm)}$defined for $f \in \mathcal{B}$ by $\left(\mathcal{F}^{( \pm)} f\right)(k)=\mathcal{F}^{( \pm)}(k) f$ is uniquely extended to a partial isometry on $L^{2}(\mathcal{M})$ with initial set $\mathcal{H}_{a c}(H)$ and final set $\widehat{\mathcal{H}}$.

(2) For $f \in D(H)$, we have

$$
\left(\mathcal{F}^{( \pm)} H f\right)(k)=k^{2}\left(\mathcal{F}^{( \pm)} f\right)(k) .
$$

(3) $\mathcal{F}^{( \pm)}(k)^{*}$ is an eigenoperator in the sense that

$$
\left(H-k^{2}\right) \mathcal{F}^{( \pm)}(k)^{*} \varphi=0, \quad \text { for all } \varphi \in \mathbf{h}_{\infty} .
$$

(4) For any $f \in \mathcal{H}_{a c}(H)$, the inversion formula holds:

$$
f=\int_{0}^{\infty} \mathcal{F}^{( \pm)}(k)^{*}\left(\mathcal{F}^{( \pm)} f\right)(k) d k .
$$

We have thus constructed a spectral representation (generalized Fourier transformation) for $H$.

Lemma 3.3. For any $k^{2} \in(0, \infty) \backslash \mathcal{E}$,

$$
\mathcal{N}(k):=\left\{u \in \mathcal{B}^{*} ;\left(-\Delta_{G}-E_{0}-k^{2}\right) u=0\right\}=\mathcal{F}^{( \pm)}(k)^{*} \mathbf{h}_{\infty} .
$$

Theorem 3.4. For any $\varphi^{(-)}=\left(\varphi_{1}^{(-)}, \cdots, \varphi_{N}^{(-)}\right) \in \mathbf{h}_{\infty}$, there exist unique $u \in$ $\mathcal{N}(k)$ and $\varphi^{(+)}=\left(\varphi_{1}^{(+)}, \cdots, \varphi_{N}^{(+)}\right) \in \mathbf{h}_{\infty}$ such that on each end $\mathcal{M}_{j}$, the asymptotic expansion

$$
u \simeq w_{j}^{(-)}(k) \varphi_{j}^{(-)}+w_{j}^{(+)}(k) \varphi_{j}^{(+)}
$$

holds. Moreover the operator

$$
\begin{aligned}
& S_{g e o}(k): \mathbf{h}_{\infty} \rightarrow \mathbf{h}_{\infty}, \\
& S_{\text {geo }}(k) \varphi^{(-)}=\varphi^{(+)}
\end{aligned}
$$

is unitary. 
3.4. Rellich's theorem, Radiation condition. In the Euclidean case, that is, for the manifold $\mathbb{R}^{n}$, there are two types of boundary conditions at infinity. A solution to the equation $\left(-\Delta_{G}-k^{2}\right) u=f, k>0$, in $\mathbb{R}^{n}$ is said to satisfy the outgoing radiation condition, if $\left(\frac{\partial}{\partial r}-i k\right) u=o\left(|x|^{-(n-1) / 2}\right)$. Similarly, $u$ is said to satisfy the incoming radiation condition, if $\left(\frac{\partial}{\partial r}+i k\right) u=o\left(|x|^{-(n-1) / 2}\right)$. Using the space $\mathcal{B}^{*}$, we can rewrite these conditions as

$$
\lim _{R \rightarrow \infty} \frac{1}{R} \int_{1<r<R}\left\|\left(\frac{\partial}{\partial r} \mp i k\right) u(r)\right\|_{L^{2}\left(S^{n-1}\right)}^{2} r^{(n-1)} d r=0
$$

Moreover, the classical Rellich theorem says that if $u \in \mathcal{B}^{*}\left(\mathbb{R}^{n}\right)$ solves a homogeneous Helmholtz equation and satisfies an incoming or outgoing radiation condition (3.10), then $u=0$.

These results can be extended to a more general case. Namely, for any $k>0$ such that $k^{2} \notin \mathcal{E}$, it is possible to define an incoming and outgoing radiation conditions for $u \in \mathcal{B}^{*}$. They are defined by

$$
\lim _{R \rightarrow \infty} \frac{1}{R} \sum_{j=1}^{N}\left(\int_{1<r<R}\left\|\left(\frac{\partial}{\partial r}-i E_{j}(k)\right) u(\cdot, r)\right\|_{L^{2}\left(M_{j}\right)}^{2} \rho_{j}(r)^{(n-1) / 2} d r\right)=0,
$$

and

$$
\lim _{R \rightarrow \infty} \frac{1}{R} \sum_{j=1}^{N}\left(\int_{1<r<R}\left\|\left(\frac{\partial}{\partial r}+i E_{j}(k)\right) u(\cdot, r)\right\|_{L^{2}\left(M_{j}\right)}^{2} \rho_{j}(r)^{(n-1) / 2} d r\right)=0,
$$

corrrespondingly. Here $E_{j}(k)$ is some operator in $L^{2}\left(M_{j}\right)$.

In typical situations, the space $\mathcal{B}^{*}$ enjoys the following property.

Theorem 3.5. Let $u \in \mathcal{B}^{*}$ satisfies $\left(-\Delta_{G}-E_{0}-\lambda\right) u=0$ for $\lambda \in(0, \infty) \backslash \mathcal{E}$ together with an outgoing (3.11), or incoming (3.12) radiation condition. Then $u=0$.

Namely, as for the decay at infinity, $\mathcal{B}^{*}$ is the smallest space for non-trivial solutions to the Helmholtz equation $\left(-\Delta_{G}-E_{0}-\lambda\right) u=0$. This is a generalization of the classical Rellich's theorem.

Theorem 3.6. A solution $u \in \mathcal{B}^{*}$ of the equation $\left(-\Delta_{G}-E_{0}-k^{2}\right) u=f \in \mathcal{B}$ satisfying either the outgoing radiation condition, or the incoming radiation condition is unique and is given by $u=R\left(k^{2}+i 0\right) f$, or $u=R\left(k^{2}-i 0\right) f$, respectively.

3.5. Inverse problem. Having completed the forward problem, we can now enter into the inverse problem. Since $\mathcal{M}$ has $N$ number of ends, the S-matrix $S_{\text {geo }}(k)$ is an $N \times N$ matrix-valued unitary operator, $S_{g e o}(k)=\left(S_{i j}(k)\right)_{i, j=1}^{N}$. We pick up the entry $S_{11}(k)$, and try to reconstruct the whole manifold $\mathcal{M}$ and the Riemannain metric from $S_{11}(k)$.

Take the end $\mathcal{M}_{1}$ and split $\mathcal{M}$ into 2 parts:

$$
\mathcal{M}=\mathcal{M}_{\text {ext }} \cup \mathcal{M}_{\text {int }}, \quad \mathcal{M}_{\text {ext }}=\mathcal{M}_{1} \cap\{r>2\}, \quad \mathcal{M}_{\text {int }}=\mathcal{M} \backslash \mathcal{M}_{\text {ext }} .
$$

Let $H_{\text {ext }}$ be the self-adjoint unbounded operator $-\Delta_{G}$ in $L^{2}\left(\mathcal{M}_{\text {ext }}\right)$ identifed by the Neumann boundary condition at $r=2$. Let $H_{\text {int }}$ be the similarly defined selfadjoint unbounded operator $-\Delta_{G}$ in $L^{2}\left(\mathcal{M}_{\text {int }}\right)$ with Neumann boundary condition 
at $r=2$. One can then solve the boundary value problem on $\mathcal{M}_{\text {ext }}$ and $\mathcal{M}_{\text {int }}$ :

$$
\begin{gathered}
\left\{\begin{array}{l}
\left(-\Delta_{G}-E_{0}-k^{2}\right) u=0, \quad \text { in } \quad \mathcal{M}_{\text {ext }}, \\
\frac{\partial u}{\partial r}=f, \quad \text { on } \quad \partial \mathcal{M}_{1}=\{r=2\} \\
u \text { satisfies the outgoing radiation condition. }
\end{array}\right. \\
\left\{\begin{array}{l}
\left(-\Delta_{G}-E_{0}-k^{2}\right) u=0, \quad \text { in } \quad \mathcal{M}_{\text {int }}, \\
\frac{\partial u}{\partial r}=f, \quad \text { on } \quad \partial \mathcal{M}_{1}=\{r=2\}, \\
u \text { satisfies the outgoing radiation condition if } \mathrm{N} \geq 2 .
\end{array}\right.
\end{gathered}
$$

Let $\Lambda_{\text {ext }}(k)$ and $\Lambda_{\text {int }}(k)$ be the associated Neumann to Dirichlet maps:

$$
\begin{aligned}
& \Lambda_{\text {ext }}(k):\left.f \rightarrow u_{e x t}\right|_{\{r=2\}}, \\
& \Lambda_{\text {int }}(k):\left.f \rightarrow u_{i n t}\right|_{\{r=2\}},
\end{aligned}
$$

$u_{\text {ext }}$ and $u_{\text {int }}$ being a unique solution to (3.14) and (3.15), respectively. Here and below, we assume that $k^{2}$ is not on the exceptional sets associated with $\mathcal{H}_{\text {ext }}$ and $\mathcal{H}_{\text {int }}$ or an eigenvalue of $H_{\text {int }}$ if $\mathcal{H}_{\text {int }}$ is a bounded region.

The following lemma is a bridge between the scattering problem and the interior boundary value problem.

Lemma 3.7. Given $\Lambda_{\text {ext }}(k)$ for all $k>0$, the $S$-matrix $S_{11}(k)$ and the $N D$ map $\Lambda_{\text {int }}(k)$ determine each other for all $k>0, k^{2} \notin \mathcal{E}$.

This is a well-known fact. The proof for the case of the Euclidean space is given in [25], and the hyperbolic space case is deal with in [26]. In the proof of this lemma, Rellich's uniqueness theorem plays a crucial role.

With the aid of Lemma 3.7, assuming that we know the end $\mathcal{M}_{1}$, we can obtain $\Lambda_{\text {int }}(k)$ for all $k \in \mathbb{C}$ from the $(1,1)$-component of the S-matrix $S_{11}(k)$ for $k>0$. One can then apply the $\mathrm{BC}$ method to $\mathcal{M}_{\text {int }}$, namely we have

Theorem 3.8. Suppose we are given two (possibly non-compact) Riemannian manifolds $\mathcal{M}_{\text {int }}^{(1)}, \mathcal{M}_{\text {int }}^{(2)}$ such that there is a diffeomorphism $\psi: \partial \mathcal{M}_{\text {int }}^{(1)} \rightarrow \partial \mathcal{M}_{\text {int }}^{(2)}$ and the Dirichlet-to-Neumann maps satisfy $\psi^{*}\left(\Lambda_{\text {int }}^{(2)}(k) f\right)=\Lambda_{\text {int }}^{(1)}(k) \psi^{*} f$ for all $k \in(a, b)$, $b>a>0$ and $f \in C_{0}^{\infty}\left(\partial \mathcal{M}_{\text {int }}^{(2)}\right)$. Then $\mathcal{M}^{(1)}$ and $\mathcal{M}^{(2)}$ are isometric.

The detailed proof of this theorem it is too long to review here but we explain below the sketch of the main steps of the proof. The details are seen in [7, 32] for the case of compact manifolds and in [27, 43] for non-compact manifolds. The first step needed to prove Theorem 3.8 is to consider the hyperbolic problem corresponding to 3.15

$$
\left\{\begin{array}{l}
\left(\partial_{t}^{2}-\Delta_{G}\right) u^{F}(x, t)=0, \quad \text { in } \quad \mathcal{M}_{\text {int }} \times \mathbb{R}_{+}, \\
\frac{\partial u^{F}}{\partial \nu}=F(x, t), \quad \text { on } \quad \partial \mathcal{M}_{\text {int }} \times \mathbb{R}_{+}, \\
\left.u^{F}(x, t)\right|_{t=0}=0,\left.\quad \partial_{t} u^{F}(x, t)\right|_{t=0}=0 .
\end{array}\right.
$$

Assume next that we are given $\partial \mathcal{M}_{\text {int }}$ and the operator $\Lambda_{\text {int }}(k)$ for $k \in(a, b)$. Since $\Lambda_{\text {int }}(k)$ is meromorphic operator depending on $k \in \mathbb{C}$, this data determine $\Lambda_{\text {int }}(k)$ for all $k \in \mathbb{C}$. Since the Fourier transform $\left(\mathcal{F}_{t \rightarrow \omega} u^{F}\right)(x, \omega)$ of $u^{F}(x, t)$ with 
respect to $t$ satisfies (3.15) with $k^{2}+E_{0}=\omega^{2}$, we can determine the hyperbolic Dirichlet-to-Neumann map $\Lambda^{(h)}:\left.F \mapsto u^{F}\right|_{\partial \mathcal{M}_{i n t} \times \mathbb{R}_{+}}$. This map can be used to compute inner products of waves.

Lemma 3.9. (Blagovestchenskii identity) Let $F, H \in C_{0}^{\infty}\left(\partial \mathcal{M}_{\text {int }} \times \mathbb{R}_{+}\right)$and $T>0$. Then

$$
\begin{gathered}
\int_{\mathcal{M}_{\text {int }}} u^{F}(x, T) u^{H}(x, T) d V_{G}(x)= \\
=\frac{1}{2} \int_{L} \int_{\partial \mathcal{M}_{i n t}}\left(F(x, t)\left(\Lambda^{(h)} H\right)(x, s)-\left(\Lambda^{(h)} H\right)(x, t) h(x, s)\right) d S_{G}(x) d t d s,
\end{gathered}
$$

where

$$
L=\{(s, t): 0 \leq t+s \leq 2 T, t<s, t, s>0\}
$$

Proof. Let

$$
w(t, s)=\int_{\mathcal{M}_{\text {int }}} u^{F}(x, t) u^{H}(x, s) d V_{G}(x) .
$$

Then, by integration by parts, we see that

$$
\begin{aligned}
\left(\partial_{t}^{2}-\partial_{s}^{2}\right) w(t, s)= & =\int_{\mathcal{M}_{\text {int }}}\left[\Delta_{G} u^{F}(x, t) u^{H}(x, s)-u^{F}(x, t) \Delta_{G} u^{H}(x, s)\right] d V_{G}(x) \\
& =\int_{\partial \mathcal{M}_{i n t}}\left[\partial_{\nu} u^{F}(t) u^{H}(s)-u^{F}(t) \partial_{\nu} u^{H}(s)\right] d S_{G}(x) \\
& =\int_{\partial \mathcal{M}_{i n t}}\left[\Lambda^{(h)} F(x, t) u^{H}(x, s)-u^{F}(x, t) \Lambda^{(h)} H(x, s)\right] d S_{G}(x) .
\end{aligned}
$$

Moreover,

$$
\left.w\right|_{t=0}=\left.w\right|_{s=0}=0,\left.\quad \partial_{t} w\right|_{t=0}=\left.\partial_{s} w\right|_{s=0}=0 .
$$

Thus, $w$ is the solution of the initial-boundary value problem for the one-dimensional wave equation in the domain $(t, s) \in[0,2 T] \times[0,2 T]$ with known source and zero initial and $\Lambda^{(h)}$. Solving this problem, we determine $w(t, s)$ in the domain where $t+s \leq 2 T$ and $t<s$. In particular, $w(T, T)$ gives the assertion.

The other result is based on the following fundamental theorem by D. Tataru [59, 60.

Theorem 3.10. Let $u=u(x, t)$ solve the wave equation $u_{t t}-\Delta_{G} u=0$ in $\mathcal{M}_{\text {int }} \times \mathbb{R}$ and $\left.u\right|_{\Gamma \times\left(0,2 T_{1}\right)}=\left.\partial_{\nu} u\right|_{\Gamma \times\left(0,2 T_{1}\right)}=0$, where $\emptyset \neq \Gamma \subset \partial \mathcal{M}_{\text {int }}$ is open. Then

$$
u(x, t)=0 \text { in } K_{\Gamma, T_{1}},
$$

where

$$
K_{\Gamma, T_{1}}=\left\{(x, t) \in \mathcal{M}_{\text {int }} \times \mathbb{R}: d(x, \Gamma)<T_{1}-\left|t-T_{1}\right|\right\}
$$

is the double cone of influence.

(The proof of this theorem, in full generality, is in [59]. A simplified proof for the considered case is in 32.)

The observability Theorem 3.10 gives rise to the following approximate controllability: 
Corollary 3.11. For any open $\Gamma \subset \partial M$ and $T_{1}>0$,

$$
\mathrm{cl}_{L^{2}\left(\mathcal{M}_{\text {int }}\right)}\left\{u^{F}\left(\cdot, T_{1}\right): F \in C_{0}^{\infty}\left(\Gamma \times\left(0, T_{1}\right)\right)\right\}=L^{2}\left(\mathcal{M}_{\text {int }}\left(\Gamma, T_{1}\right)\right) .
$$

Here

$$
\mathcal{M}_{\text {int }}\left(\Gamma, T_{1}\right)=\left\{x \in \mathcal{M}_{\text {int }}: d(x, \Gamma)<T_{1}\right\}=K_{\Gamma, T_{1}} \cap\left\{t=T_{1}\right\}
$$

is the domain of influence of $\Gamma$ at time $T_{1}$ and $L^{2}\left(\mathcal{M}_{\text {int }}\left(\Gamma, T_{1}\right)\right)=\left\{a \in L^{2}\left(\mathcal{M}_{\text {int }}\right)\right.$ : $\left.\operatorname{supp}(a) \subset \mathcal{M}_{\text {int }}\left(\Gamma, T_{1}\right)\right\}$.

Lemma 3.12. Let $T>0$ and $\Gamma_{j} \subset \partial \mathcal{M}_{\text {int }}, j=1, \ldots, J$, be non-empty, relatively compact open sets, $0 \leq T_{j}^{-}<T_{j}^{+} \leq T$. Assume we are given $\partial \mathcal{M}_{\text {int }}$ and the response operator $\Lambda^{(h)}$. This data determines the inner product

$$
J_{\mathcal{N}}^{T}\left(F_{1}, F_{2}\right)=\int_{\mathcal{N}} u^{F_{1}}(x, t) u^{F_{2}}(x, t) d V_{G}(x)
$$

for given $t>0$ and $F_{1}, F_{2} \in C_{0}^{\infty}\left(\partial \mathcal{M}_{\text {int }} \times \mathbb{R}_{+}\right)$, where

$$
\mathcal{N}=\bigcap_{j=1}^{J}\left(\mathcal{M}_{i n t}\left(\Gamma_{j}, T_{j}^{+}\right) \backslash \mathcal{M}_{\text {int }}\left(\Gamma_{j}, T_{j}^{-}\right)\right) \subset \mathcal{M}_{\text {int }} .
$$

We give the proof of Lemma 3.12 only for the case when $J=1$ and $T_{1}^{-}=0$ so that $\mathcal{N}=\mathcal{M}_{\text {int }}\left(\Gamma_{1}, T_{1}^{+}\right)$. We note that when the manifold $\partial \mathcal{M}_{\text {int }}$ and the response operator $\Lambda^{(h)}$ are given, we can determine the metric $G_{\partial \mathcal{M}_{i n t}}$ of the boundary $\partial \mathcal{M}_{\text {int }}$. We say that the manifold $\partial \mathcal{M}_{\text {int }}$ with its metric and the response operator $\Lambda^{(h)}$ form the boundary data.

Consider first the case $F_{1}=F_{2}=F$. Let $\left.B=\Gamma_{1} \times\left[T-T_{1}^{+}, T\right]\right)$. For all $H \in C_{0}^{\infty}(B)$ it holds that $\operatorname{supp}\left(u^{H}(\cdot, T)\right) \subset \mathcal{N}$, and thus

$$
\begin{aligned}
& \left\|u^{F}(T)-u^{H}(T)\right\|_{L^{2}\left(\mathcal{M}_{i n t}\right)}^{2} \\
& =\int_{\mathcal{N}}\left(u^{F}(x, T)-u^{H}(x, T)\right)^{2} d V_{G}(x)+\int_{\mathcal{M}_{\text {int }} \backslash \mathcal{N}}\left(u^{F}(x, T)\right)^{2} d V_{G}(x) .
\end{aligned}
$$

Let $\chi_{\mathcal{N}}(x)$ be the characteristic function of the set $\mathcal{N}$. By Corollary 3.11, there is $H \in C_{0}^{\infty}(B)$ such that the norm $\left\|\chi_{\mathcal{N}} u^{F}(T)-u^{H}(T)\right\|_{L^{2}\left(M, d V_{\mu}\right)}$ is arbitrarily small. This shows that $J_{\mathcal{N}}^{T}\left(F_{1}, F_{2}\right)$ can be found by

$$
J_{\mathcal{N}}^{T}(F, F)=\left\|u^{F}(T)\right\|_{L^{2}\left(\mathcal{M}_{\text {int }}\right)}^{2}-\inf _{H \in C_{0}^{\infty}(B)} \mathcal{F}(H),
$$

where

$$
\mathcal{F}(H)=\left\|u^{F}(T)-u^{H}(T)\right\|_{L^{2}\left(\mathcal{M}_{\text {int }}\right)}^{2} .
$$

Since $\mathcal{F}(H)$ can be computed when the boundary data is given by Lemma 3.9] it follows that we can determine $J_{\mathcal{N}}^{T}(F, F)$ for any $F \in C_{0}^{\infty}\left(\partial M \times \mathbb{R}_{+}\right)$. Now, since

$$
J_{\mathcal{N}}^{T}\left(F_{1}, F_{2}\right)=\frac{1}{4}\left(J_{\mathcal{N}}^{T}\left(F_{1}+F_{2}, F_{1}+F_{2}\right)-J_{\mathcal{N}}^{T}\left(F_{1}-F_{2}, F_{1}-F_{2}\right)\right),
$$

Lemma 3.12 follows in the case when $J=1$ and $T_{1}^{-}=0$.

To reconstruct $\left(\mathcal{M}_{\text {int }}, G\right)$, we use a special representation, the boundary distance representation, $R\left(\mathcal{M}_{\text {int }}\right)$, of $\mathcal{M}_{\text {int }}$ and show that the boundary data determine $R\left(\mathcal{M}_{\text {int }}\right)$. Consider a map $R: \mathcal{M}_{\text {int }} \rightarrow C\left(\partial \mathcal{M}_{\text {int }}\right)$,

$$
R(x)=r_{x}(\cdot) ; \quad r_{x}(z)=d_{g}(x, z), z \in \partial \mathcal{M}_{\text {int }},
$$


i.e., $r_{x}(\cdot)$ is the distance function from $x \in \mathcal{M}_{\text {int }}$ to the points on $\partial \mathcal{M}_{\text {int }}$. The image $R\left(\mathcal{M}_{\text {int }}\right) \subset C\left(\partial \mathcal{M}_{\text {int }}\right)$ of $R$ is called the boundary distance representation of $\mathcal{M}_{\text {int }}$. The set $R\left(\mathcal{M}_{\text {int }}\right)$ is a metric space with the distance inherited from $C\left(\partial \mathcal{M}_{\text {int }}\right)$ with the standard norm $\|f\|_{\infty}=\max _{z \in \partial \mathcal{M}_{\text {int }}}|f(z)|$ of $C\left(\partial \mathcal{M}_{\text {int }}\right)$. We denote this distance by $d_{C}$. The map $R$, due to the triangular inequality, is Lipschitz,

$$
d_{C}\left(r_{x}, r_{y}\right) \leq d_{g}(x, y)
$$

Note that if $\partial \mathcal{M}_{\text {int }}$ is unbounded, which corresponds to the possible case of unbounded $M_{i}, r_{x}(\cdot)$ becomes unbounded and, instead of the distance $d_{C}\left(r_{x}, r_{y}\right)$, it is convenient to use a modified one

$$
d_{b}\left(r_{x}, r_{y}\right)=\sup _{z \in \partial \mathcal{M}_{i n t}}\left(\frac{\left|r_{x}(z)-r_{y}(z)\right|}{1+\left|r_{x}(z)-r_{y}(z)\right|}\right) .
$$

When $x, y$ run over a compact in $\mathcal{M}_{i n t}, d_{b}\left(r_{x}, r_{y}\right)$ is equivalent to $d_{C}\left(r_{x}, r_{y}\right)$.

The map $R: \mathcal{M}_{\text {int }} \rightarrow R\left(\mathcal{M}_{\text {int }}\right) \subset C\left(\partial \mathcal{M}_{\text {int }}\right)$ is an embedding. Many results of differential geometry, such as Whitney or Nash embedding theorems, concern the question how an abstract manifold can be embedded to some simple space, such as a higher dimensional Euclidean space. In the inverse problem we need to construct a "copy" of the unknown manifold in some known space, and as we assume that the boundary is given, we do this by embedding the manifold $\mathcal{M}_{\text {int }}$ to the known, although infinite dimensional function space $C\left(\partial \mathcal{M}_{\text {int }}\right)$.

The basic observation needed to construct $R\left(\mathcal{M}_{\text {int }}\right)$ is that the set $\mathcal{N}$ given in (3.21) has non-zero measure if and only if

$$
P\left(\left\{\left(\Gamma_{j}, T_{j}^{+}, T_{j}^{-}\right): j=1, \ldots, J\right\}\right)=\sup _{f} J_{N}^{T}(f, f),
$$

is non-zero.

Theorem 3.13. Let $\left\{z_{n}\right\}_{n=1}^{\infty}$ be a dense set on $\partial \mathcal{M}_{\text {int }}$ and $r(\cdot) \in C\left(\partial \mathcal{M}_{\text {int }}\right)$ be an arbitrary continuous function. Then $r \in R\left(\mathcal{M}_{\text {int }}\right)$ if and only if for all $L \in \mathbb{N}_{+}$, it holds that

$$
P\left(\left\{\left(z_{j}, r\left(z_{n}\right)+\frac{1}{L}, r\left(z_{n}\right)-\frac{1}{L}\right) ; j=1, \ldots, L\right\}\right)>0 .
$$

Moreover, condition (3.25) can be verified when the manifold $\partial \mathcal{M}_{\text {int }}$ and the response operator $\Lambda^{(h)}$ are given. Hence the pair $\left(\partial \mathcal{M}_{i n t}, \Lambda^{(h)}\right)$ determines uniquely the boundary distance representation $R\left(\mathcal{M}_{\text {int }}\right)$ of $\left(\mathcal{M}_{\text {int }}, G\right)$.

The idea of to proof Theorem 3.13 is that we see that the set $\left\{x \in \mathcal{M}_{\text {int }} ; \mid d\left(z_{j}, x\right)-\right.$ $r\left(z_{n}\right) \mid<\frac{1}{L}$ for all $\left.j=1, \ldots, L\right\}$ contains a point $x_{0}$ for all $L$ if and only if $r_{x_{0}}(z)=$ $r(z)$ for all $z \in \partial \mathcal{M}_{\text {int }}$.

Next let us consider a compact manifold $\left(\mathcal{M}_{\text {int }}, G\right)$ that is geodesically regular manifold, i.e.,

i) For any $x, y \in \mathcal{M}_{\text {int }}$ there is a unique geodesic $\gamma$ joining these points.

ii) Any geodesic $\gamma([a, b])$ can be continued to a geodesic $\gamma\left(\left[a^{\prime}, b^{\prime}\right]\right)$ whose endpoints lie on the boundary.

Consider $R\left(\mathcal{M}_{\text {int }}\right)$ as a metric space $\left(R\left(\mathcal{M}_{\text {int }}\right), d_{\infty}\right)$ with the distance inherited from $L^{\infty}\left(\partial \mathcal{M}_{\text {int }}\right), d_{\infty}\left(r_{x}, r_{y}\right)=\left\|r_{x}-r_{y}\right\|_{L^{\infty}\left(\partial \mathcal{M}_{\text {int }}\right)}$. Then the mapping $R$ is an isometry, i.e.

$$
d_{\infty}\left(r_{x}, r_{y}\right)=d(x, y)
$$


Indeed, let $\gamma([a, b])$ be the shortest geodesic from $y$ to $x$. Continue this geodesic to the shortest geodesic $\gamma\left(\left[a^{\prime}, b\right]\right)$, where $z=\gamma\left(a^{\prime}\right) \in \partial \mathcal{M}_{\text {int }}$. Then

$$
r_{x}(z)-r_{y}(z)=\left|\gamma\left(\left[a^{\prime}, b\right]\right)\right|-\left|\gamma\left(\left[a^{\prime}, a\right]\right)\right|=b-a=d(x, y) .
$$

Hence,

$$
d(x, y) \leq d_{\infty}\left(r_{x}, r_{y}\right) .
$$

The opposite inequality is valid by the triangular inequality, yielding that $d(x, y)=$ $d_{\infty}\left(r_{x}, r_{y}\right)$. This means that, if we know a priori that $\left(\mathcal{M}_{i n t}, G\right)$ is geodesically regular, then $\left.\left(R\left(\mathcal{M}_{\text {int }}\right), d_{\infty}\right)\right)$ is isometric to $\left(\mathcal{M}_{\text {int }}, G\right)$ and the problem of the reconstruction of $\left(\mathcal{M}_{\text {int }}, G\right)$ from the data $\left(\partial \mathcal{M}_{\text {int }}, \Lambda^{(h)}\right)$ is solved. The above ideas can be generalized to prove Theorem 3.8 .

Let us note that only one diagonal entry $S_{11}^{(2)}(k)$ is necessary to recover the whole manifold using Lemma 3.7 and Theorem 3.8. Therefore, our final inverse procedure is as follows.

Theorem 3.14. Suppose we are given two non-compact Riemannian manifolds $\mathcal{M}^{(1)}, \mathcal{M}^{(2)}$ having $N_{1}$ and $N_{2}$ numbers of ends, respectively. Let $\mathcal{M}_{1}^{(1)}$ and $\mathcal{M}_{1}^{(2)}$ be their first ends, and suppose that there exists $r_{0}>1$ such that $\mathcal{M}_{1}^{(1)} \cap\left\{r>r_{0}\right\}$ and $\mathcal{M}_{1}^{(2)} \cap\left\{r>r_{0}\right\}$ are isometric. Assume furthermore that their $(1,1)$-entries of $S$-matrices coincide : $S_{11}^{(1)}(k)=S_{11}^{(2)}(k)$ for all $k>0, k^{2} \notin \mathcal{E}^{(1)} \cup \mathcal{E}^{(2)}$. Then $\mathcal{M}^{(1)}$ and $\mathcal{M}^{(2)}$ are isometric.

\section{EXAMPLes}

4.1. Model metric. Spectral properties of $-\Delta_{G}$ depends largely on the growth of the manifold $\mathcal{M}$ at infinity. Looking at an end $(1, \infty) \times M$, we pick up here 4 basic examples, and examine what is going on. We have so far studied the following cases:

(1) $G=(d r)^{2}+e^{2 r} h$,

(2) $G=(d r)^{2}+r^{2} h$,

(3) $G=(d r)^{2}+h$,

(4) $G=(d r)^{2}+e^{-2 r} h$,

where $h$ is a metric on $M$.

These are well-known classical examples, and have some distinguished properties.

4.2. Tools for the resolvent estimates. Before going into the details, we explain here the method for resolvent estimates. There are several ways of proving LAP for Laplacians on manifolds. One is E. Mourre's abstract commutator theory [51, another is Melrose' theory of scattering metric 48, 49. Our method is different from both of them. We employ the classical Eidus' approach of integration by parts 14. Given the equation $\left(-\Delta_{G_{0}}-E_{0}-z\right) u=f$ on the model space $\mathcal{M}_{0}=I_{0} \times M_{0}$, we expand $u$ by the eigenvectors of $-\Delta_{M_{0}}$. The problem is then reduced to the 1dimensional case. The main step is to multiply this 1-dimensional equation by (the derivative of ) solution and integrate by parts to obtain some identities. Standard arguments from perturbation theory then prove the necessary resolvent estimates. See e.g. 28], Lemmas 2.4, 2.5, Theorem 2.7, or [27, Lemmas $2.4 \sim 2.8$. This method is no less powerful than the above two machineries, and gives us results by elementary computation. All of the above 4 examples are treated by this method. 
4.3. Euclidean metric. Let us start with the Euclidean metric (2). In this case, we take $\mathcal{M}_{0}=\mathbb{R}^{n}$, and the perturbed metric $g_{i j} d x^{i} d x^{j}$ is assumed to satisfy

$$
\partial_{x}^{\alpha}\left(g_{i j}(x)-\delta_{i j}\right)=O\left(|x|^{-1-|\alpha|-\epsilon_{0}}\right), \quad \text { for all } \alpha \text { and some } \epsilon_{0}>0 .
$$

There are plenty of papers dealing with scattering in the asymptotically Euclidean space, and all the requisites are prepared in e.g. 27] or 61. Consulting them, one can see that the arguments in $\S 3$ work well, and Theorem 3.14 holds. In this case, there are no embedded eigenvalues in $(0, \infty)$. The radiation condition is the standard one:

$$
\lim _{R \rightarrow \infty} \frac{1}{R} \int_{|x|<R}\left|\left(\frac{\partial}{\partial r} \mp i k\right) u\right|^{2} d x=0 .
$$

4.4. Cylindrical ends. Let us consider (3). In the daily life example, it arises as the problem of waveguide. As a model space, we take $M_{0}$ to be a compact $(n-1)$-dimensional manifold without boundary. Let $\mathcal{M}_{0}=(1, \infty) \times\left(M_{0}, h\right)$, and $\Delta_{0}=\Delta_{\left(M_{0}, h\right)}$. The perturbed metric is assumed to satisfy

$$
\left|\partial_{X}^{\alpha}\left(g_{i j}(X)-h_{i j}(x)\right)\right| \leq C_{\alpha}(1+r)^{-1-\epsilon}, \quad \text { for all } \alpha \text { and some } \epsilon>0
$$

where $x$ denotes the variable in $M_{0}$, and $X=(r, x)$.

The waveguide has many features different from the conical Euclidean metric (2). In the first place, it may have eigenvalues embedded in the continuous spectrum. The 2 nd feature is that the scattering in the waveguide has many channels. Let $\left\{\lambda_{m}\right\}_{m=1}^{\infty}$ be the eigenvalues of $-\Delta_{0}$. Then, since $-\Delta_{G}$ has the form

$$
-\Delta_{G}=-\left(\frac{\partial}{\partial r}\right)^{2}-\Delta_{0},
$$

when the total system has energy $\lambda>0$, only the states with $\lambda_{m} \leq \lambda$ occur in the scattering phenomena. The radiation condition is thus defined by

$$
\lim _{R \rightarrow \infty} \frac{1}{R} \int_{1<r<R}\left\|\left(\frac{\partial}{\partial r} \mp i \sqrt{\lambda+\Delta_{0}}\right) u(r, \cdot)\right\|_{L^{2}\left(M_{0}\right)}^{2} d r=0 .
$$

Because of this channel property, the scattering matrix changes its size according to the energy, higher the energy, bigger the size of the S-matrix.

To discuss the inverse scattering, we assume that one end is strictly cylindrical. Namely, the end $\mathcal{M}_{1}$ is equal to $[1, \infty) \times M_{0}$ with metric $(d r)^{2}+h$. We take an artificial boundary $r=2$ in $\mathcal{M}_{1}$, and split $\mathcal{M}$ into the exterior and interior domains. As in $\S 3$, we derive interior N-D map from the knowledge of $(1,1)$-entry of the S-matrix. However, the physical S-matrix is not sufficient to determine the interior N-D map. The key observation here is that the physical S-matrix $S_{11}(k)$ admits an analytic continuation into the upper (physical) half plane, and, down to the real axis, determines non-physical scattering matrix, which is defined using the exponentially growing solutions of the reduced wave equation instead of the physical plane wave. Therefore Theorem 3.14 holds in this case.

The exponentially growing solution is the crucial idea found by Faddeev in his multi-dimensional inverse scattering theory, as well as Calderón's inverse boundary value problem. What is interesting is that this apparently artificial exponentially growing solutions appear naturally in the waveguide problem. The details are given in $[28$.

We can also allow the ends of type (2) and (3) at the same time. Namely, if our ends $\mathcal{M}_{1}, \cdots, \mathcal{M}_{N}$ are composed of two parts: $\mathcal{M}_{1}, \cdots, \mathcal{M}_{\mu}$, which are Euclidean 
ends, and $\mathcal{M}_{\mu+1}, \cdots, \mathcal{M}_{N}$, which are cylindrical ends, the results in subsections 4.3, 4.4 are applied as well.

4.5. Hyperbolic space. We turn to the case (1). The problem here is that in dimensions $\geq 3$, the infinity of hyperbolic manifold may have a complicated structure. Therefore, we restrict ourselves to the simple case, and as a model space, we take $\mathcal{M}_{0}=M_{0} \times(0, \infty)$ equipped with the metric $\left((d y)^{2}+h(x, d x)\right) / y^{2}$, where $M_{0}$ is a compact manifold equipped with metric $h(x, d x)$. This model has two infinities, $y=0$ corresponding to the infinite volume, which we call regular end, and $y=\infty$ corresponding to cusp. We take $H_{0}=-\Delta_{\mathcal{M}_{0}}-(n-1)^{2} / 4$. Then $\sigma\left(H_{0}\right)=[0, \infty)$. We split $\mathcal{H}_{0}$ into two parts : $\{0<y<1\}$ and $\{y>1\}$. By the change of variable $y=e^{-r}$, the former becomes a model space for (1). The change of variable $y=e^{r}$ makes the latter to a model space for (4).

The perturbed metric is assumed to have the form

$$
\begin{gathered}
d s^{2}=y^{-2}\left((d y)^{2}+h(x, d x)+A(x, y, d x, d y)\right) \\
A(x, y, d x, d y)=\sum_{i, j=1}^{n-1} a_{i j}(x, y) d x^{i} d x^{j}+2 \sum_{i=1}^{n-1} a_{i n}(x, y) d x^{i} d y+a_{n n}(x, y)(d y)^{2},
\end{gathered}
$$

where $a_{i j}(x, y)$ satisfies, for some $\epsilon_{0}>0$

$$
\left|\widetilde{D}_{x}^{\alpha} D_{y}^{\beta} a(x, y)\right| \leq C_{\alpha \beta}(1+|\log y|)^{-\min (|\alpha|+\beta, 1)-1-\epsilon_{0}}, \quad \text { for all } \quad \alpha, \beta .
$$

Here, $D_{y}=y \partial y, \widetilde{D}_{x}=\widetilde{y}(y) \partial_{x}, \widetilde{y}(y) \in C^{\infty}((0, \infty)), \widetilde{y}(y)=y$ for $y>2, \widetilde{y}(y)=1$ for $0<y<1$.

If one of the ends is a regular infinity, there are no embedded eigenvalues. If all the ends are cusps, there may be embedded eigenvalues. The radiation condition is formulated as

$$
\begin{gathered}
\lim _{R \rightarrow \infty} \frac{1}{\log R} \int_{1 / R<y<1}\left\|\left(D_{y}-i \sigma_{ \pm}(k)\right) u(y)\right\|_{L^{2}\left(M_{0}\right)}^{2} \frac{d y}{y^{n}}=0, \\
\lim _{R \rightarrow \infty} \frac{1}{\log R} \int_{1<y<R}\left\|\left(D_{y}-i \sigma_{\mp}(k)\right) u(y)\right\|_{L^{2}\left(M_{0}\right)}^{2} \frac{d y}{y^{n}}=0, \\
\sigma_{ \pm}(k)=\frac{n-1}{2} \mp i k .
\end{gathered}
$$

Note that these are standard radiation conditions

(3.11) and (3.12) since $r=|\log y|$.

The inverse scattering from regular ends works well as in $\S 3$. Namely, suppose we are given two such asymptotically hyperbolic manifolds $\mathcal{M}^{(1)}, \mathcal{M}^{(2)}$ whose regular ends $\mathcal{M}_{1}^{(1)}$ and $\mathcal{M}_{1}^{(2)}$ are isometric, and the $(1,1)$ components of the S-matrix coincide for all $k$. Then $\mathcal{M}^{(1)}$ and $\mathcal{M}^{(2)}$ are isometric. This is proven in 27.

We remark that Sá Barreto [57, using the framework of scattering metric due to Melrose, proved the existence of isometry between two asymptotically hyperbolic manifolds having the same scattering matrix without the assumption that $\mathcal{M}_{1}^{(1)}$ and $\mathcal{M}_{1}^{(2)}$ are isometric, although the decay assumption at infinity are different from ours. In [22, it is extended to asymptotically hyperbolic complex manifolds. 
4.6. Poisson integral and the space $\mathcal{B}^{*}$. Before entering into the problem of cusp, we briefly look at some aspects of Poisson integral. Let us take the most basic example of Poincaré disc : $D=\{z \in \mathbb{C} ;|z|<1\}$. It is well-known that the Poisson integral

$$
u(z)=\frac{1}{2 \pi} \int_{0}^{2 \pi}\left(\frac{1-|z|^{2}}{\left|e^{i \theta}-z\right|^{2}}\right)^{s} f(\theta) d \theta
$$

$f(\theta)$ being a function on the boundary $\partial D=S^{1}$, is a solution to the equation

$$
\left(-\Delta_{G}-4 s(1-s)\right) u=0
$$

for all $s \in \mathbb{C}$. Our space $\mathcal{B}^{*}$, for which $f(\theta) \in L^{2}\left(S^{1}\right)$, has the following special feature : $\left\{u \in \mathcal{B}^{*} ;\left(-\Delta_{G}-\frac{1}{4}-k^{2}\right) u=0\right\}$ is the smallest space of solutions as regard to decay at infinity. In fact, the Rellich type theorem says that the solution of (4.3) decaying faster than the elements of $\mathcal{B}^{*}$ vanishes identically. Although this space is the smallest, it contains sufficiently many solutions for the inverse scattering. The largest solution space was found by Helgason 23, namely all solutions of (4.3) are represented as (4.2), where $f(\theta)$ is Sato's hyperfunction. This theorem was extended to the general symmetric spaces by Kashiwara-Kowata-MinemuraOkamoto-Oshima-Tanaka 34. The theorem of Helgason suggests that one may control the solution space of the equation $\left(-\Delta_{G}-\frac{1}{4}-k^{2}\right) u=0$ through function spaces on the boundary at infinity. This enables us to extend the notion of S-matrix. We utilize this idea for the cusp.

\section{Arithmetic surface and generalized S-matrix}

We restrict ourselves here to the 2-dimensional case. Lots of examples of hyperbolic surfaces are given by the action of discrete groups on the upper half plane. If we consider the geometrically finite case, which implies that the associated quotient space is a polygon with sides of finite number of geodesics, the infinity consists of a finite number of cusp and funnel (the latter being a slightly perturbed regular infinity discussed in 4.5). However, if the Fuchsian group contains elliptic elements, the quotient space $\mathcal{M}$ has singularities. Note that $\mathcal{M}$ itself is an analytic manifold without singularities, however, the hyperbolic metric induced from $\mathbb{C}_{+}$becomes singular at elliptic fixed points. Thus they are orbifolds, moreover, many examples of quotient spaces appearing in number theory have only cusp at infinity. They are non-compact, but has a finite volume. The result of the previous section cannot be applied to this case. As a matter of fact, the S-matrix at a cusp end does not have enough information to reconstruct the metric, since the cusp gives only onedimensional contribution to the continuous spectrum. A remedy lies in generalizing the notion of S-matrix.

Assume that $\mathcal{M}_{1}=(1, \infty) \times(-1 / 2,1 / 2)$ with two sides identified. Take any solution $u$ of the equation $\left(H-k^{2}\right) u=0$, and expand it into the Fourier series on $\mathcal{M}_{1}$ :

$$
u(x, y)=\sum_{n \in \mathbf{Z}} e^{2 \pi n x} u_{n}(y) .
$$

Then $u_{n}$ satisfies

$$
y^{2}\left(-\partial_{y}^{2}+(2 n y)^{2}\right) u_{n}(y)-\frac{1}{4} u_{n}(y)=k^{2} u_{n}(y),
$$


hence is written as

$$
u_{n}(y)=\left\{\begin{array}{l}
\widetilde{a}_{n} y^{1 / 2} I_{-i k}(2 \pi|n| y)+\widetilde{b}_{n} y^{1 / 2} K_{i k}(2 \pi|n| y), \quad n \neq 0, \\
a_{0} y^{1 / 2-i k}+b_{0} y^{1 / 2+i k}, \quad n=0 .
\end{array}\right.
$$

where $I_{\nu}$ and $K_{\nu}$ are modified Bessel functions. We then see that all solutions of the equation $\left(H-k^{2}\right) u=0$ behave like

$$
u(x, y) \sim a_{0} y^{1 / 2-i k}+\sum_{n \neq 0} a_{n} e^{i n x+|n| y}+b_{0} y^{1 / 2+i k}+\sum_{n \neq 0} b_{n} e^{i n x-|n| y},
$$

as $y \rightarrow \infty$. Given an exponentially growing wave represented by the first two terms in (5.1), one can uniquely construct a solution of $\left(H-k^{2}\right) u=0$ belonging to $\mathcal{B}^{*}$ on ends $\mathcal{M}_{j}, j \neq 1$, and behaving like (5.1) in $\mathcal{M}_{1}$. We call the mapping

$$
\mathcal{S}_{11}(k):\left\{a_{n}\right\}_{n \in \mathbf{Z}} \rightarrow\left\{b_{n}\right\}_{n \in \mathbf{Z}}
$$

the generalized $S$-matrix. Passing to the Fourier series, we see that to define (5.2), we are using a class of analytic functionals bigger than Sato's hyperfunction. Note that $\mathcal{S}_{11}(k)$ is an infinite matrix, and the usual S-matrix $S_{11}(k)$ is its $(0,0)$-entry. This generalized S-matrix has enough information for the inverse scattering. We make an artificial boundary in $\mathcal{M}_{1}$ and consider the boundary value problem in the interior domain. Then the knowledge of $\mathcal{S}_{11}(k)$ for all $k$ enables us to determine the N-D map $\Lambda_{\text {int }}(k)$, hence to apply the BC method.

In the interior domain $\mathcal{M}_{\text {int }}$, there is a finite number singular points. Since they have a special structure, we can deal with $\mathcal{M}_{\text {int }}$ as a manifold with conical singularities, to which we can apply the BC method as well. We can thus prove the following theorem.

Theorem 5.1. Suppose we are given two 2-dimensional asymptotically hyperbolic surfaces with conical singularities $\mathcal{M}^{(1)}, \mathcal{M}^{(2)}$. Suppose they have pure cusp ends $\mathcal{M}_{1}^{(1)}, \mathcal{M}_{1}^{(2)}$, i.e. with metric $\left((d y)^{2}+(d x)^{2}\right) / y^{2}$, and their generalized $S$-matrices coincide : $\mathcal{S}_{11}^{(1)}(k)=\mathcal{S}_{11}^{(2)}(k)$, for all $k$. Then $\mathcal{M}^{(1)}$ and $\mathcal{M}^{(2)}$ are isometric.

In particular, if $\mathcal{H}^{(i)}=\Gamma^{(i)} / \mathbf{H}^{2}$ with geometrically finite Fuchsian group $\Gamma^{(i)}$, and their generalized S-matrix coincide, $\Gamma^{(1)}$ and $\Gamma^{(2)}$ are conjugate. See [29] for details. Also note that the results in subsections 4.5 and 5.1 also hold when $\mathcal{M}$ has both regular ends and cusps.

As in the previous cases, the key idea for the proof of Theorem 5.1 is the use of exponentially growing solutions of the Helmholtz equation, which appears naturally here because of the form of hyperbolic metric. For cylindrical ends, we have encountered a similar situation, in which case, however, the non-physical scattering matrix is obtained by the analytic continuation of the physical scattering matrix. This is not true for the cusp. In fact, Zelditch 62 constructed an example of non-isometric hyperbolic surfaces with the same physical scattering matrix.

\section{WORKS IN PROGRESS}

6.1. Higher dimensional asymptotically hyperbolic orbifolds. In higer dimensions, we have new phenomenon in the problem in $\S 5$. Consider the case of $n=3$, and let the Picard group $\Gamma=S L(2, \mathbf{Z}+i \mathbf{Z})$ act on $\mathbf{H}^{3}$ through quarternions. The resulting quotient space is a 3-dimensional hyperbolic manifold with singularities, i.e. an orbifold. Unlike the 2-dimensional case, the singularities are not 
confined in a compact set. They form an unbounded curve, and the manifold at infinity is not a smooth manifold, but a 2-dimensional orbifold (see [15]). However, the results for the forward problem also hold in this case with no essential change, and we are also expecting the inverse scattering results as well.

The structure of the manifold at infinity of general hyperbolic manifold is complicated (see e.g. 24]). The forward and inverse scattering for this general case are challenging problems.

6.2. Intermediate metrics. We have worked on the problems (1)-(4), however, there is no reason that we must restrict ourselves to these cases. There is a wide area of problems for the intermediate metrics between (1) and (4), and those outside. For example, 40, 31 are dealing with non-existence of embedded eigenvalues, and 41 studies the LAP. We expect that the inverse scattering theory can also be developed in these cases.

\section{REFERENCES}

[1] S. Agmon, Spectral theory of Schrödinger operators on Euclidean and non-Euclidean spaces, Comm. Pure Appl. Math. 39, Supplement (1986), S 3 - S 16.

[2] M. Anderson, A. Katsuda, Y. Kurylev, M. Lassas, and M. Taylor, Boundary regularity for the Ricci equation, Geometric Convergence, and Gel'fand's Inverse Boundary Problem, Inventiones Mathematicae 158 (2004), 261-321

[3] M. I. Belishev, An approach to multidimensional inverse problems for the wave equation, Dokl. Akad. Nauk SSSR 297 (1987), 524-557.

[4] M. I. Belishev, Boundary control in construction of manifolds and metrics (the BC method), Inverse Problems 13 (1997), R1-R45.

[5] M. I. Belishev and A. S. Blagovestcenskii, Multidimensional analogs of the Gel'fand-LevitanKrein equations in inverse problems for the wave equations, Ill-posed problems of Mathematical Physics and Analysis, Nobosibirsk : Nauk (1992), 50-63.

[6] M. I. Belishev, A nonstationary inverse problem for the multidimensional wave equation in the large, ZAP. Nauchn. Sem. LOMI 165 (1987), 21-30.

[7] M. I. Belishev and Y. V. Kurylev, To the reconstruction of a Riemannian manifold via its spectral data (BC method), Comm. in P. D. E. 17 (1992), 767-804.

[8] K. Bingham, Y. Kurylev, M. Lassas and S. Siltanen, Iterative time reversal control for inverse problems. Inverse Problems and Imaging 2 (2008), 63-81.

[9] A. S. Blagovestcenskii, The local method of solution of the non-stationary inverse scattering problem for an inhomogeneous string, Trudy Mat. Inst. Steklova 115 (1971), 28-38.

[10] A. S. Blagovestcenskii, The nonselfadjoint inverse matrix boundary problem for a hyperbolic differential equation, in Problems of Mathematical Physics, 5, Spectral Theory, Izdat. Leningrad Univ., Leningrad (1971), 38-62.

[11] D. Borthwick, Spectral Theory of Infinite-Area Hyperbolic Surfaces, Progress in Mathematics 256, Birkhäuser, Boston-Basel-Berlin (2007).

[12] D. Borthwick, C. Judge and P. Perry, Selberg's zeta function and the spectral geometry of geometrically finite hyperbolic surfaces, Comment. Math. Helv. 80 (2005), 483-515.

[13] T. Christiansen, Sojourn times, manifolds with infinite cylindrical ends, and inverse problem for planer waveguides, J. Anal. Math. 107 (2009), 79-106.

[14] D. M. Eidus, The principle of limit amplitude, Russ. Math. Survey 24 (1969), 97-167.

[15] J. Elstrodt, F. Grunewald and J. Mennicke, Groups Acting in Hyperbolic Spaces, Springer, Berlin-Heidelberg-New York (1998).

[16] L. D. Faddeev, Inverse problem of quantum scattering theory, J. Sov. Math. 5 (1976), 334-396.

[17] I. M. Gel'fand and B. M. Levitan, On the determination of a differential equation from its spectral function, Izv. Akad. Nauk USSR, Ser. Mat. 15 (1951), 309-360.

[18] A. Greenleaf, M. Lassas, and G. Uhlmann, On nonuniqueness for Calderon's inverse problem, Math. Res. Lett. 10 (2003), 685-693.

[19] A. Greenleaf, Y. Kurylev, M. Lassas, and G. Uhlmann, Full-wave invisibility of active devices at all frequencies. Commun. in Math. Phys. 275 (2007), 749-789 
[20] A. Greenleaf, Y. Kurylev, M. Lassas, and G. Uhlmann, Invisibility and Inverse Problems. Bulletin of the Amer. Math. Soc. 46 (2009), 55-97.

[21] A. Greenleaf, Y. Kurylev, M. Lassas, and G. Uhlmann:, Cloaking Devices, Electromagnetic Wormholes and Transformation Optics. SIAM Review 51 (2009), 3-33.

[22] C. Guillarmou and A. Sá Barreto, Scattering and inverse scattering on ACH manifolds, J. reine angew. Math. 622 (2008), 1-55.

[23] S. Helgason, A duality for symmetric spaces with applications to group representations, Adv. in Math. 5 (1970), 1-154.

[24] P. D. Hislop, The geometry and spectra of hyperbolic manifolds, Proc. Indian Acad. Sci. (Math. Sci.) 104 (1994), 715-776.

[25] V. Isakov and A. Nachman, Global uniqueness for a two-dimensional semilinear elliptic inverse problem, Trans. Amer. Math. Soc., 347 (1993), 3375-3390.

[26] H. Isozaki, Inverse spectral problems on hyperbolic manifolds and their applications to inverse boundary value problems in Euclidean space, Amer. J. Math. 126 (2004), 1261-1313.

[27] H. Isozaki and Y. Kurylev, Introduction to spectral theory and inverse problems on asymptotically hyperbolic manifolds, arXiv 1102.5382.

[28] H. Isozaki, Y. Kurylev and M. Lassas, Forward and inverse scattering problem on manifolds with asymptotically cylindrical ends, Journal of Funct. Anal. 258 (2010), 2060-2118.

[29] H. Isozaki, Y. Kurylev and M. Lassas, Conic singularities, generalized scattering matrix and inverse scattering on asymptotically hyperbolic surfaces, arXiv:1108.1577

[30] H. Isozaki, Inverse spectral theory, in Topics in the Theory of Schrödinger Operators, edit. H. Araki and H. Ezawa, World Scientific (2003), 93-143.

[31] K. Ito and E. Skibsted, Absence of embedded eigenvalues for Riemannian Laplacians, arXiv 1109.1928.

[32] A. Katchalov, Y. Kurylev and M. Lassas, Inverse Boundary Spectral Problems, Chapman and Hall/CRC, Monographs and Surveys in Pure and Applied Mathematics, 123 (2001).

[33] A. Katchalov, Y. Kurylev, M. Lassas, and N. Mandache, Equivalence of time-domain inverse problems and boundary spectral problem, Inverse problems 20 (2004), 419-436

[34] M. Kashiwara, A. Kowata, K. Minemura, K. Okamoto, T. Oshima, M. Tanaka, Eigenfunctions of invariant differential operators on a symmetric spaces, Ann. Math. 107 (1978), 1-39.

[35] M. Kerker, Invisible bodies, J. Opt. Soc. Am. 65 (1975), 376.

[36] G. M. Khenkin and R. G. Novikov, The $\bar{\partial}$ equation in the multi-dimensional inverse scattering problem, Russian Math. Surveys, 42 (1987), 109-180.

[37] M. G. Krein, Determination of the density of an inhomogeneous string from its spectrum, Dokl. Akad. Nauk SSSR 76 (1951), 345-348.

[38] M. G. Krein, On inverse problems for an inhomogeneous string, Dokl. Akad. Nauk SSSR 82 (1951), 669-672.

[39] K. Krupchyk, Y. Kurylev, M. Lassas, Inverse spectral problems on a closed manifold. Journal de Mathematique Pures et Appliquees 90 (2008), 42-59.

[40] H. Kumura, The radial curvature of an end that makes eigenvalues vanish in the essential spectrum, I, Math. Ann. 346 (2010), 795-828.

[41] H. Kumura, Limiting absorption principle on manifolds having ends with various measure growth rate limits, arXiv. math DG/0606125.

[42] Y. Kurylev, M. Lassas, T. Yamaguchi. Uniqueness and stability in inverse spectral problems for collapsing manifolds, arxiv.math: 1209.587v2

[43] M. Lassas, M. Salo, G. Uhlmann, Wave imaging. Chapter in Handbook of Mathematical Methods in Imaging, O. Scherzer (Ed.), Springer-Verlag, 2011, 50 pp

[44] M. Lassas, M. Taylor and G. Uhlmann, The Dirichlet-to-Neumann map for complete Riemannian manifolds with boundary, Comm. in Anal. and Geom. 11 (2003), 207-222.

[45] M. Lassas and G. Uhlmann, On determining a Riemannian manifold from the Dirichlet-toNeumann map, Annales Scientifiques de l' Ecole Normale Superiéure, 34 (2001), 771-787.

[46] V. A. Marchenko, The construction of the potential energy from the phases of the scattering waves, Dokl. Akad. Nauk SSSR 104 (1955), 695-698.

[47] R. Mazzeo and R. Melrose, Meromorphic extension of the resolvent on complete spaces with asymptotically constant negative curvature, J. Funct. Anal. 75 (1987), 260-310.

[48] R. Mazzeo and A. Vasy, Analytic continuation of the resolvent of the Laplacian on symmetric spaces of noncompact type, J. Funct. Anal. 228 (2005), 311-368.

[49] R. Melrose, Geometric Scattering Theory, Cambridge University Press (1995). 
[50] R. Melrose, G. Uhlmann, Generalized Backscattering and the Lax-Phillips Transform, Serdica Math. J. 34 (2008), 1026-1044.

[51] E. Mourre, Absence of singular continuous spectrum for certain self-adjoint operators, Comm. in Math. Phys. 78 (1981), 391-400.

[52] W. Müller, Manifolds with cusp of rank one, spectral theory and $L^{2}$-index theorem, Lecture Notes in Math. 1224, Springer (1987).

[53] A. Nachman, Reconstruction from boundary measurements, Ann. of Math. 143 (1988), 531576.

[54] L. Päivärinta, M. Salo, and G. Uhlmann, Inverse scattering for the magnetic Schrödinger operator, J. Funct. Anal. 259 (2010), 1771-1798.

[55] P. A. Perry, The spectral geometry of geometrically finite hyperbolic manifolds, Spectral Theory and Mathematical Physics: A Festschrift in Honor of Barry Simon's 60th Birthday, Proc. Sympos. Pure Math., 76, A. M. S. Providence, RI (2007), 289-328.

[56] M. Reed and B. Simon, Methods of Modern Mathematical Physics, III : Scattering Theory, Academic Press (1979).

[57] A. Sá Barreto, Radiation fields, scattering and inverse scattering on asymptotically hyperbolic manifolds, Duke Math. J. 129 (2005), 407-480.

[58] J. Sylvester and G. Uhlmann, A global uniqueness theorem for an inverse boundary value problem, Ann. of Math. 125 (1987), 153-169.

[59] D. Tataru, Unique continuation for solutions to PDEs; between Hörmander's theorem and Holmgren's theorem, Comm. in PDE. 20 (1995), 855-884.

[60] D. Tataru, On the regularity of boundary traces for the wave equation. Ann. Scuola Norm. Sup. Pisa Cl. Sci. 26 (1998), 185-206.

[61] D. R. Yafaev, Mathematical Scattering Theory, Analytic Theory, Mathematical Surveys and Monographs 158, A. M. S., Providence, Rhode Island (2009).

[62] S. Zelditch, Kuznecov sum formulae and Szegö limit formulae on manifolds, Commun. in P. D. E. 17 (1992), 221-260.

Hiroshi Isozaki, Institute of Mathematics, University of Tsukuba, Tsukuba, 305-8571, Japan; Yaroslav Kurylev, Department of Mathematics, University College of London, United Kingdom; Matti Lassas, Department of Mathematics and Statistics, University OF Helsinki, Finland

E-mail address: isozakih@math.tsukuba.ac.jp,y.kurylev@ucl.ac.uk,mjl@rni.helsinki.fi 